\title{
CONTROL DE FORMACIONES DE VEHÍCULOS MARINOS DE SUPERFICIE CON RESTRICCIONES DE ENTRADA
}

\author{
Jesús M. de la Cruz García \\ Dpt. Arquitectura Computadores y Automática. Facultad de Ciencias Físicas, UCM \\ jmcruz@ucm.es \\ José A. López Orozco \\ Dpt. Arquitectura Computadores y Automática. Facultad de Ciencias Físicas, UCM \\ jalopez@ucm.es \\ Eva Besada Portas \\ Dpt. Arquitectura Computadores y Automática. Facultad de Ciencias Físicas, UCM \\ ebesada@ucm.es \\ Joaquin Aranda Almansa \\ Dpt. Informática y Automática. E.T.S. Informática, UNED \\ jaranda@dia.uned.es
}

\begin{abstract}
Resumen
Se presenta un algoritmo para el control de formaciones y seguimiento de caminos para vehículos no holónomos con aplicación a vehículos marinos de superficie. El algoritmo sigue una estructura líder-seguidor y es una extensión de un artículo anterior [1] a curvas parametrizadas, y no sólo para caminos formados por tramos rectos y circulares. Además, se tiene en cuenta las restricciones que restringen las posibles trayectorias que pueden ser seguidos por el líder. El algoritmo sigue manteniendo la ventaja de la sencillez y sólo necesita de la sintonía de un parámetro para cada uno de los vehículos. Se muestran experimentos simulados con modelos reales a escala que validan el método propuesto.
\end{abstract}

Palabras Clave: control de formaciones, seguimiento de caminos, vehículos marinos de superficie, control de barcos.

\section{INTRODUCCIÓN}

El control de formaciones de vehículos autónomos es un área de investigación de gran importancia en la robótica móvil. Con ello se consigue resolver problemas que no pueden resolverse sólo con un robot móvil, a la vez que se logra mayor robustez ante posibles fallos de alguno de los componentes, y mayor efectividad en tiempo y coste.

En el campo de los vehículos autónomos marinos de superficie (USVs) las formaciones y colaboración de tienen importancia en aplicaciones tanto militares como civiles. Misiones de exploración, búsqueda y rescate en el mar son claros ejemplos de ello. En algunos casos es necesario que los vehículos se muevan manteniendo una formación. Ejemplos son el arrastre de una red, o barrera, para recogida de vertidos contaminantes, plásticos u otros productos, y el reabastecimiento de un barco por otro mientras navegan.

También se prevé en un futuro la cooperación de redes de vehículos heterogéneos de tierra mar y aire, para recogida de datos que permitan el mejor conocimiento del entorno marino para un adecuado aprovechamiento de los recursos y para mejorar el conocimiento del estado medioambiental de los mares y del propio planeta. En estos escenarios, el seguimiento de un USV de otros vehículos de superficie o submarinos se prevé esencial dadas su capacidades como interfaces para actuar de repetidores de comunicaciones entre vehículos submarinos y aéreos o espaciales.

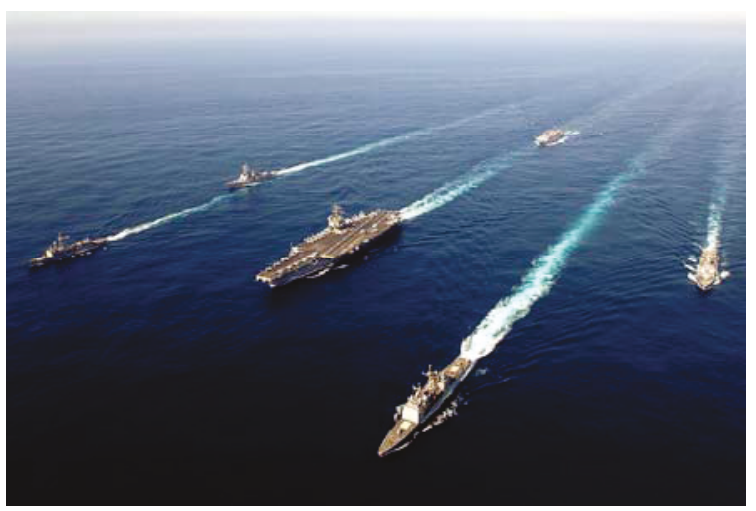

Figura 1. Formación con seguimiento de un líder. Wikipedia. 
En [2]-[3] se da una visión general de USVs desarrollados, de las tendencias de diseño y de la investigación básica y los problemas que se plantean.

Revisiones sobre la coordinación y cooperación de vehículos se pueden encontrar en [4]-[6]. Se han propuesto distintos marcos o esquemas de control de formaciones entre ellas: métodos basados en el comportamiento, estructuras virtuales, técnicas de potencial de campo, método líder-seguidor [7]-[14]. Esta última es especialmente atractiva por su simplicidad y escalabilidad [8]; además, el método incluye el problema del seguimiento de un vehículo por otro [4],[9],[16]. A partir de los trabajos señalados y sus referencias se puede conseguir una amplia visión de la evolución y estado actual del tema.

En este trabajo se hace una extensión del algoritmo de [1] para el control de formaciones con el método líder-seguidor. En [1] el método se aplicaba a trayectorias compuestas de tramos rectos y circulares, $\mathrm{y}$ aunque en muchos casos la trayectoria de los vehículos se describe de este modo, se extiende al seguimiento de cualquier curva paramétrica que pueda ser físicamente seguida por el líder y el seguidor. Una trayectoria compuesta de tramos rectos y circulares es un caso particular de curva paramétrica a trozos y cae dentro de la nueva solución. Además, se dan las restricciones que debe cumplir la trayectoria para que el líder y el seguidor puedan realizarla. El método puede también ser utilizado para que un vehículo autónomo siga a otro vehículo que no sigue un camino determinado sino que, por ejemplo, la trayectoria es la que decide el capitán en cada momento.

El método [1] tiene la ventaja, frente a otros métodos, de su sencillez y que sólo requiere un parámetro de sintonía con una clara significación física. La extensión realizada mantiene ambas propiedades y se apoya en el trabajo [8] para vehículos no holónomos, en el cual se dan condiciones necesarias y suficientes en los límites de las velocidades del líder y del seguidor para que estos mantengan la formación independientemente de la trayectoria del líder. En ambos trabajos la posición del seguidor con respecto al líder se da en términos de una distancia y un ángulo deseados entre el seguidor y el líder. En [8] el ángulo está restringido a tener un valor absoluto menor de pi medios, y se muestra que la posición del seguidor no es fija con respecto al sistema de referencia ligado al líder sino que varía en arcos de circunferencia adecuados centrados en el sistema de referencia del líder. En el trabajo se introduce por primera vez en la literatura el concepto de formación estacionaria con el fin de fijar la posición del seguidor con respecto al líder. Esta posición se puede ver como perteneciente a una cierta circunferencia concéntrica con la circunferencia definida por la curvatura del líder, en todos los casos en que la curvatura del líder es distinta de cero. Además, en el método propuesto se elimina la restricción en el ángulo entre el seguidor y el líder, de modo que el seguidor puede hacerlo con cualquier ángulo entre menos pi y pi.

La organización del trabajo es como sigue. En la sección 2 se hace un resumen de los resultados de [1] y [8]. A continuación, en la sección 3 , se introduce el concepto de formación estacionaria y se demuestra su validez para todo ángulo $\phi \in[-\pi, \pi]$. En la sección 4 presenta el método propuesto. En la sección 5 se muestran resultados experimentales de validación obtenidos con modelos de buques. En la sección 6 se dan las conclusiones.

Aunque se dispone de las demostraciones, no se han incluido en el trabajo por falta de espacio y serán publicadas en un próximo trabajo.

\section{RESULTADOS PREVIOS}

Introducimos la terminología y los resultados de los trabajos [8] y [1] necesarios para la mejor comprensión del método propuesto. En primer lugar introducimos los de la primera referencia.

Consideramos que la dinámica del líder y el seguidor están dadas por

$$
\begin{aligned}
& \dot{x}_{i}=v_{i}(t) \cos \left(\psi_{i}(t)\right) \\
& \dot{y}_{i}=v_{i}(t) \sin \left(\psi_{i}(t)\right) \\
& \dot{\psi}_{i}(t)=\omega_{i}(t)
\end{aligned}
$$

Usaremos $i=L$ para hacer referencia la líder, e $i=F$ para el seguidor. Las señales de control para el seguidor son las velocidades lineales y angulares: $v_{F}(t), \omega_{F}(t)$. Si $v_{i}(\mathrm{t}) \neq 0$; ponemos $\kappa_{i}(t)=\omega_{i}(t) / v_{i}(t)$, que es la curvatura del camino seguida por el vehículo $i$ en el instante $t$. Representamos mediante $P_{i}(t)=\left(x_{i}(t), y_{i}(t)\right)^{\mathrm{T}}$ la posición de $i$ en $t, \psi_{i}(\mathrm{t}) \mathrm{su}$ rumbo, $\tau\left(\psi_{i}(t)\right)=\left[\cos \left(\psi_{i}(t)\right), \sin \left(\psi_{i}(t)\right)\right]^{\mathrm{T}}$ el vector velocidad normalizado, y $v\left(\psi_{i}(t)\right)\left[-\sin \left(\psi_{i}(t)\right)\right.$, $\left.\cos \left(\psi_{i}(t)\right)\right]^{\mathrm{T}} \quad$ el vector normalizado ortogonal a $\tau\left(\psi_{i}(t)\right)$; así, $R\left(\psi_{i}(t)\right)=\left\{\tau\left(\psi_{i}(t)\right), v\left(\psi_{i}(t)\right)\right\}$ representa el sistema de referencia ligado al robot en el instante $t$, a la vez que representa también la matriz de rotación que transforma los ejes cuerpo a los ejes inerciales, o que aplica las coordenadas inerciales a coordenadas cuerpo. Un robot $\boldsymbol{R}$ está definido por el vector $(x, y$, $\psi)^{\mathrm{T}}$ y por el control $(\nu, \omega)^{\mathrm{T}}$ con dinámica (1).

La formación deseada se especifica por $(d, \phi)$ siendo $d$ la distancia deseada entre el seguidor y el líder, y $\phi$ el ángulo deseado entre el seguidor y el líder visto desde el sistema de referencia del seguidor, figura1. 


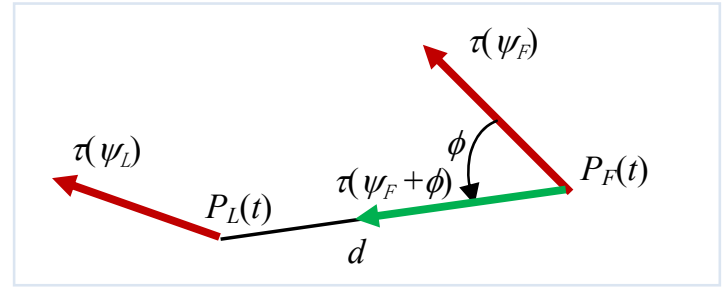

Figura 1: Formación $(d, \phi)$

Definición 1. Sean los robots $\boldsymbol{R}_{\boldsymbol{L}}=\left(P_{L}^{T}, \psi_{L}\right)^{\mathrm{T}}, \boldsymbol{R}_{\boldsymbol{F}}=$ $\left(P_{F}^{T}, \psi_{F}\right)^{\mathrm{T}}$, y $d>0, \quad \phi \in(-\pi / 2, \pi / 2)$. Decimos que $\boldsymbol{R}_{\boldsymbol{L}}$ y $\boldsymbol{R}_{\boldsymbol{F}}$ están

i. en formación $(d, \phi)$ con líder $\boldsymbol{R}_{\boldsymbol{L}}$ en el instante $t$ si se verifica (2)

ii. en formación $(d, \phi)$ con líder $\boldsymbol{R}_{\boldsymbol{L}}$ si se verifica (2) para $\mathrm{t} \geq 0$;

$$
P_{L}(t)=P_{F}(t)+d \tau\left(\psi_{F}(t)+\phi\right)
$$

En [8] se demuestra que la posición que define la posición del seguidor no es fija con respecto al sistema de referencia ligado al líder, sino que varía en arcos de circunferencia centrados en el sistema de referencia ligado al líder; así, mientras que la distancia $d$ se mantiene fija, el ángulo $\psi_{F}$ puede variar.

Definición 2. Sea $\boldsymbol{R}$ un robot y $\left(V, K^{+}, K^{-}\right), V>0$, $K^{+} \geq 0, K^{-} \leq 0$. Decimos que $\boldsymbol{R}$ satisface la restricción $\left(V, K^{+}, K^{-}\right)$, si para todo $t \geq 0,0<v(t) \leq V, K^{-} \leq \kappa(t)$ $\leq K^{+}$donde $\kappa(t)$ es la curvatura del camino seguido por $\boldsymbol{R}$ y $v(t)$ su velocidad.

La siguiente suposición da una restricción física en todos los robots que se consideran.

Suposición 1. Sean $V_{p}, K_{p}^{-}, K_{p}^{+}$tres constantes. Supondremos que todo robot sigue la siguiente restricción en su trayectoria $\left(V_{p}, K_{p}^{-}, K_{p}^{+}\right)$.

En [8] se da un teorema que da condiciones necesarias y suficientes en los límites de las velocidades del líder y del seguidor para la existencia de una ley de control que permite que el seguidor mantenga la formación independientemente de la trayectoria del líder. El teorema se resume en las siguientes propiedades cuando dos robots $\boldsymbol{R}_{L}, \boldsymbol{R}_{\boldsymbol{F}}$ están en formación $(d, \phi)$, y el robot $\boldsymbol{R}_{\boldsymbol{L}}$ satisface en su trayectoria la restricción $\left(V_{0}, K_{0}^{-}, K_{0}^{+}\right)$y el robot $\boldsymbol{R}_{F}$ tiene la restricción $\left(V_{p}, K_{p}^{-}, K_{p}^{+}\right)$y como ley de control

$$
v_{F}(t)=\frac{v_{L}(t) \cos \left(\psi_{L}(t)-\psi_{F}(t)-\phi\right)}{\cos (\phi)}
$$

$$
\omega_{F}=v_{L}(t) \frac{\sin \left(\psi_{L}(t)-\psi_{F}(t)\right)}{d \cos (\phi)}
$$

Propiedades:

$$
\begin{gathered}
-\frac{1}{d} \leq K_{0}^{-} \leq K_{0}^{+} \leq \frac{1}{d \cos \phi}, \quad \text { if } \phi \geq 0 \\
-\frac{1}{d \cos \phi} \leq K_{0}^{-} \leq K_{0}^{+} \leq \frac{1}{d}, \quad \text { if } \phi<0 \\
\tilde{K}_{0}^{-} \leq K_{0}^{-} \leq K_{0}^{+} \leq \tilde{K}_{0}^{+} \\
\tilde{K}_{0}^{ \pm}=\left(\operatorname{sign} K_{p}^{ \pm}\right)\left(\left(\left(K_{p}^{ \pm}\right)^{-1}-d \sin \phi\right)^{2}+D^{2} \cos ^{2} \phi\right)^{-1 / 2} \\
V_{0} \cos \left(\delta_{\phi}\right) \leq V_{p} \cos \phi, \\
\delta_{\phi}=\min \left\{0, \arcsin \left(K_{0}^{+} d \cos \phi\right)-\phi, \phi-\arcsin \left(K_{0}^{+} d \cos \phi\right)\right\}
\end{gathered}
$$

Las ecuaciones (3) dan la ley de control que hace que, si se parte de unas condiciones iniciales adecuadas, el seguidor esté en formación con el líder. Las ecuaciones (4)-(6) dan las condiciones que ligan los valores de $(d, \phi)$ válidos de acuerdo con las limitaciones físicas de los robots y la trayectoria a seguir.

Presentamos a continuación el algoritmo de control de formaciones de [1].

En [1] se da una ley de control para líder basada en seguir un punto virtual que recorre la trayectoria deseada a una distancia especificada $L$ que es el único parámetro de sintonía del algoritmo. La ley está descrita en [17] y combina los algoritmos de guiado de [18] y [19].

El seguidor utiliza una ley de control similar al líder. Se supone un punto virtual sobre la trayectoria, recta o circunferencia, que debería seguir el seguidor si estuviera en formación con el líder pero a una distancia $L$ por delante de él. La ley de control utiliza dicho punto virtual para determinar los valores $\left(v_{F}\right.$, $\left.\omega_{F}\right)^{\mathrm{T}}$. la figura 2 muestra las relaciones geométricas de la posición del vehículo seguidor $P(t)$, la posición $P_{F}(t)$, que debería tener si estuviera en formación con el líder, y la posición del punto virtual $M(t)$ situado a una distancia $L$ por delante de $P_{F}(t)$ sobre la trayectoria que se está siguiendo, que en el gráfico es una circunferencia de radio $1 / \kappa_{F}(t)$.

Se definen los siguientes ángulos: $\eta$ es el ángulo creado por la línea que une el punto virtual $M(t)$ con $P(t)$ y el vector $\tau\left(\psi_{P}\right) ; \beta$ es el ángulo que forma la línea que une los dos puntos anteriores con $\tau\left(\psi_{M}\right) ; \theta$ es el ángulo que forma $\tau\left(\psi_{M}\right)$ con $\tau\left(\psi_{P}\right)$. Claramente 


$$
\eta=\beta+\theta
$$

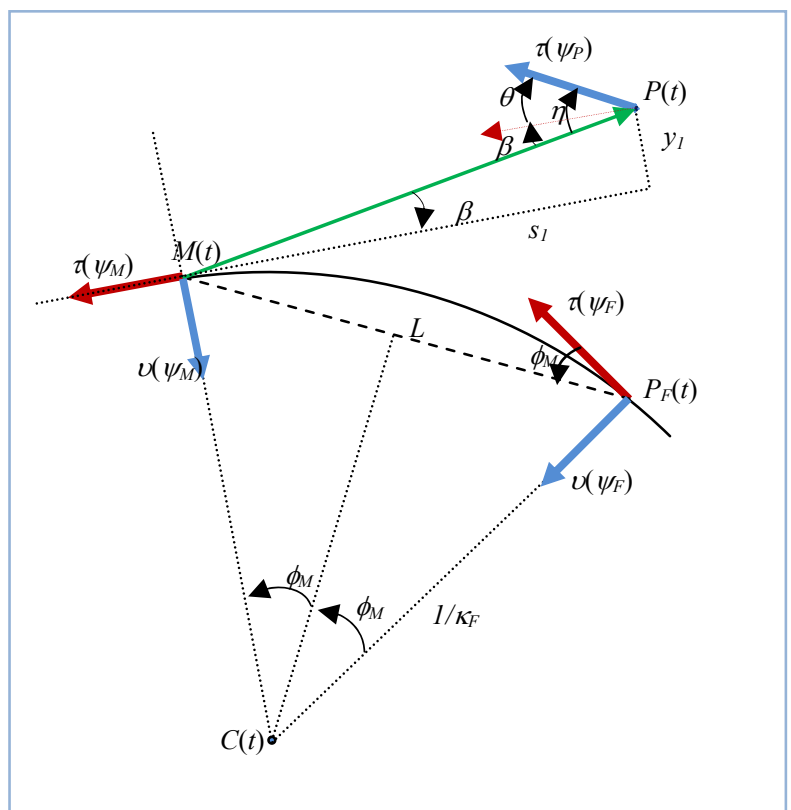

Figura 2. Geometría del seguidor, el punto virtual y la posición deseada del seguidor.

Como se ve, es necesario reconstruir la trayectoria que sigue el robot líder para situar el punto virtual. La ley de control para el vehículo seguidor es

$$
\begin{gathered}
\dot{\psi}_{P}=-\frac{2 v_{P}}{L} \sin \eta, \quad|\eta| \leq \frac{\pi}{2} \\
\dot{\psi}_{P}=-\frac{2 v_{P}}{L} \operatorname{sign} \eta, \quad|\eta|>\frac{\pi}{2} \\
v_{P}=v_{M}-K\left(s_{1}+L \cos \left(\phi_{M}\right)\right) \operatorname{sign}(\cos \theta)
\end{gathered}
$$

Las ecuaciones (8) hacen que el robot seguidor tienda a la posición lateral que debería tener para estar en formación; la ecuación (9) trata de que se mantenga a la distancia $L$ del punto virtual $M$. La velocidad de este es

$$
v_{M}=v_{L} \frac{\kappa_{L}}{\kappa_{F}}
$$

El parámetro $K$ no es libre, sino que está relacionado con la velocidad del líder, $L$ y $\phi_{M}$, [17].

\section{FORMACIÓN ESTACIONARIA}

Como hemos visto, la solución de [8] no da un único punto solución a un formación especificada $(d, \phi)$. Nos proponemos definir un punto solución único, que denominaremos formación estacionaria, que será utilizado para definir la posición única que en todo momento debería tener el seguidor. Usaremos este punto para definir un punto virtual a partir del cual se deducirá la ley de control para el vehículo seguidor. El ángulo se podrá definir en Con tal fin, definimos

$$
\psi(t) \triangleq \psi_{L}(t)-\psi_{F}(t), t \in[0, \infty)
$$

Usando (3b) se tiene

$\dot{\psi}(t)=\omega_{L}(t)-\omega_{F}(t)=\frac{v_{L}(t)}{d \cos \phi}\left(\kappa_{L}(t) d \cos \phi-\sin \psi(t)\right)$

Siguiendo a [8], si $\left|\kappa_{L}(t)\right| d \leq 1 \forall t \geq 0$, con condiciones iniciales adecuadas la solución estacionaria es

$$
\psi(t)=\arcsin \left(\kappa_{L}(t) d \cos \phi\right)
$$

Si sustituimos (12) en (3b) se tiene $\omega_{L}(t)=\omega_{F}(t)$. Además, si suponemos que $\omega_{L}(t)=\omega_{F}(t)$ a partir de (3b) se deduce la relación (12), luego la ecuación (12) se verifica si y sólo si $\omega_{L}(t)=\omega_{F}(t)$. La ecuación (12) fija entonces el siguiente ángulo para que el seguidor esté en formación

$$
\psi_{F}(t)=\psi_{L}(t)-\arcsin \left(\kappa_{L}(t) d \cos \phi\right)
$$

Nos centramos en esta posición para determinar la ley de control del seguidor.

Debido a los resultados previos, introducimos la definición de formación estacionaria.

Definición 3. Sean $\boldsymbol{R}_{L}=\left(P_{L}^{T}, \psi_{L}\right)^{\mathrm{T}}$ y $\boldsymbol{R}_{F}=\left(P_{F}^{T}, \psi_{F}\right)^{\mathrm{T}}$ be two robots, $d>0, \quad \phi \in[-\pi, \pi]$. El líder y el seguidor están:

i. En una formación estacionaria $(d, \phi)$ en el instante si están en formación $(d, \phi)$ en $t$ y sus velocidades angulares son iguales.

$$
\omega_{L}(t) \triangleq \dot{\psi}_{L}(t)=\dot{\psi}_{F}(t) \triangleq \omega_{F}(t) .
$$

ii. En una formación estacionaria $(d, \phi)$ si están en formación $(d, \phi)$ y sus velocidades angulares son iguales $\omega_{L}(t) \triangleq \dot{\psi}_{L}(t)=\dot{\psi}_{F}(t) \triangleq \omega_{F}(t)$ para $t \geq 0$.

Daremos condiciones necesarias y suficientes para la formación estacionaria. Primero consideramos $|\phi|<$ $\pi / 2$ y luego extenderemos el resultado a $|\phi|=\pi / 2$ y finalmente a $|\phi|>\pi / 2$.

\subsection{CARACTERIZACIÓN DE FORMACIÓN ESTACIONARIA}

Teorema 1. Si dos robots $\boldsymbol{R}_{L}=\left(P_{L}{ }^{T}, \psi_{L}\right)^{\mathrm{T}}$ y $\boldsymbol{R}_{F}=$ $\left(P_{F}^{T}, \psi_{F}\right)^{\mathrm{T}}$ están en una formación estacionaria $(d, \phi)$ con líder $\boldsymbol{R}_{L}, d>0$ and $|\phi|<\pi / 2$, entonces $P_{F}(\mathrm{t})$ está dado por 


$$
P_{F}(t)=P_{L}(t)-d \tau\left(\psi_{F}(t)+\phi\right)
$$

donde

$$
\psi(t) \triangleq \psi_{L}(t)-\psi_{F}(\mathrm{t})=\arcsin \left(\kappa_{L}(t) d \cos \phi\right)
$$

Además, si $\kappa_{L}(t) \neq 0$ entonces $P_{F}(t)$ es un punto en una circunferencia de centro $C(t)$, curvatura $\kappa_{F}(t), \mathrm{y}$ desplazamiento angular $\psi(t)$ con respecto a $\psi_{L}(t)$, donde (ver Figura 3$)^{1}$

$$
\begin{aligned}
& P_{F}(t)=C(t)-\frac{1}{\kappa_{F}(t)} v\left(\psi_{F}(t)\right) \\
& C(t)=P_{L}(t)+\frac{1}{\kappa_{L}(t)} v\left(\psi_{L}(t)\right) \\
& \kappa_{F}=\kappa_{L} \frac{\cos \phi}{\cos (\psi-\phi)} .
\end{aligned}
$$

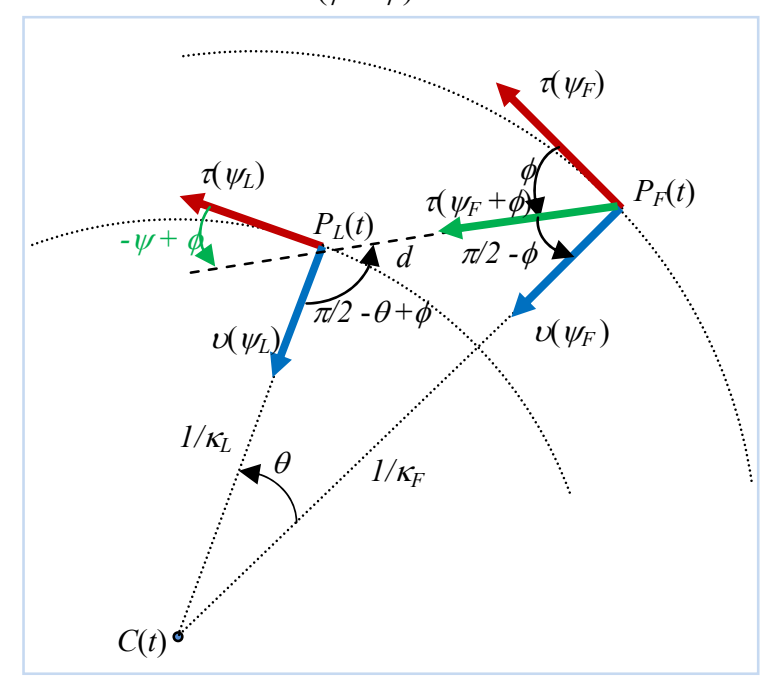

Figura 3. Formación estacionaria con $\omega_{L}>0$

Notar que la ecuación (3a) da valores iguales a la proyecciones de los dos robots de los vectores velocidad sobre la línea que une $P_{F}(t)$ y $P_{L}(t)$, por ello, la distancia entre ambos puntos permanece constante. La ecuación (3b) en el caso estacionario da velocidades angulares iguales para los extremos de los extremos del segmento $P_{F}(t) P_{L}(t)$, por lo tanto el ángulo permanece constante. De este modo, si las velocidades lineales y angulares de ambos robots permanecieran constantes describirían dos circunferencias concéntricas con radios $1 /\left|\kappa_{L}(t)\right|$, $1 /\left|\kappa_{F}(t)\right|$ si $\quad \kappa_{L}(t) \neq 0$, y seguirían líneas rectas paralela si $\kappa_{L}(t)=0$.

\footnotetext{
${ }^{1}$ No se incluyen las demostraciones de los teoremas que pueden deducirse aplicando conceptos básicos de geometría.
}

Las propiedades (4), (5) y (6) de la curvatura de la trayectoria, $d, \phi$ y la máxima velocidad puede deducirse de las propiedades geométricas del triángulo formado por $P_{L} C P_{F}$.

\subsection{EXTENSIÓN A $\phi= \pm \pi / 2$}

Aunque el teorema 1 se cumple sólo para $|\phi|<\pi / 2$ se puede extender a cualquier ángulo $\frac{\pi}{2} \leq|\phi| \leq \pi$. Primero presentamos la extensión a $|\phi|=\pi / 2$.

Teorema 2. Si dos robots $\boldsymbol{R}_{\boldsymbol{L}}=\left(P_{L}{ }^{T}, \psi_{L}\right)^{\mathrm{T}}$ y $\boldsymbol{R}_{\boldsymbol{F}}=$ $\left(P_{F}^{T}, \psi_{F}\right)^{\mathrm{T}}$ están en formación estacionaria $(d, \phi)$ con líder $\quad \boldsymbol{R}_{\boldsymbol{L}}$ que satisface las restricciones $\left(V_{0}, K_{0}^{-}, K_{0}^{+}\right)$, y $d>0,|\phi|=\pi / 2$, entonces $P_{F}(t)$ está dado por

$$
P_{F}(t)=P_{L}(t)-d \tau\left(\psi_{F}(t)+\phi\right)
$$

donde $\psi_{F}(t)=\psi_{L}(t)$. Además, si $\kappa_{L}(t) \neq 0$ entonces $P_{F}(t)$ es un punto de una circunferencia $C(t)$, curvatura $\kappa_{F}(t)$ donde

$$
\begin{aligned}
& P_{F}(t)=C(t)-\frac{1}{\kappa_{F}(t)} v\left(\psi_{F}(t)\right) \\
& C(t)=P_{L}(t)+\frac{1}{\kappa_{L}(t)} v\left(\psi_{L}(t)\right) \\
& \kappa_{F}=\frac{\kappa_{L}}{1+\operatorname{sign}(\phi) d \kappa_{L}}
\end{aligned}
$$

Se verifican las siguientes propiedades

$$
\begin{gathered}
-\frac{1}{d}<K_{0}^{-}, \quad K_{0}^{+}<\frac{1}{d} \\
\frac{1}{\frac{1}{K_{p}^{-}}-d} \leq K_{0}^{-}, \quad K_{0}^{+} \leq \frac{1}{\frac{1}{K_{p}^{+}}+d} \\
V_{0}\left(1+d K_{0}^{\max }\right) \leq V_{p} \\
\text { con } K_{0}^{\max }=\max \left\{K_{0}^{+},\left|K_{0}^{-}\right|\right\} .
\end{gathered}
$$

\subsection{EXTENSIÓN A $\pi \geq|\phi|>\pi / 2$}

Mostramos ahora que la formación estacionaria se puede extender a $\pi \geq|\phi|>\pi / 2$.

Teorema 3. Si $\boldsymbol{R}_{L}$ y $\boldsymbol{R}_{F}$ son dos robots con líder $\boldsymbol{R}_{L}=$ $\left(P_{L}(t)^{\mathrm{T}}, \psi_{L}(t)\right)$ y seguidor $\boldsymbol{R}_{F}=\left(P_{F}(t)^{\mathrm{T}}, \psi_{F}(t)\right)$ en formación estacionaria $(d, \phi)$ con $|\phi|<\pi / 2$ entonces existe otro robot $\boldsymbol{R}_{2}=\left(P_{2}(t)^{\mathrm{T}}, \psi_{2}(t)\right)$ en formación estacionaria

a) $(d, \pi-\phi)$ con líder $R_{L}$ si $\phi \geq 0$, 
b) $\quad(d,-\pi-\phi)$ con líder $R_{L}$ si $\phi<0$

donde

$$
\begin{aligned}
& P_{2}(t)=P_{L}(t)-d \tau\left(\psi_{2}-\phi\right) \\
& \psi_{2}=\psi_{L}+\arcsin \left(\kappa_{L} d \cos \phi\right) \\
& \omega_{2}=\omega_{F}, \quad v_{2}=v_{F} .
\end{aligned}
$$

\subsection{DISEÑO DE UN ROBOT EN ESTADO ESTACIONARIO}

Usamos los resultados de los teoremas 1 y 2 para dado un robot $\boldsymbol{R}_{\boldsymbol{L}}$ que satisface las restricciones $\left(V_{0}\right.$, $\left.K_{0}^{-}, K_{0}^{+}\right), d>0$ y $|\phi| \leq \pi / 2$ construir un robot en formación estacionaria $(d, \phi)$ con el líder $\boldsymbol{R}_{L}$.

Teorema 4. Sea $\boldsymbol{R}_{\boldsymbol{L}}=\left(P_{L}{ }^{T}, \psi_{F}\right)^{\mathrm{T}}$ un robot que satisface las restricciones $\left(V_{0}, K_{0}^{-}, K_{0}^{+}\right)$y $d>0$ y $|\phi|$ $\leq \pi / 2$, y que verifica (4-6) si $|\phi|<\pi / 2$, y (21-23) si $|\phi|$ $=\pi / 2$, entonces podemos construir un robot $\boldsymbol{R}_{F}=$ $\left(P_{F}^{T}, \psi_{F}\right)^{\mathrm{T}}$ que está en formación estacionaria $(d, \phi)$ con el líder $\boldsymbol{R}_{L}$ si elegimos

$$
\begin{gathered}
P_{F}(t)=P_{L}(t)-d \tau\left(\psi_{F}+\phi\right) \\
\psi=\psi_{L}-\psi_{F}=\arcsin \left(\kappa_{L} d \cos \phi\right) .
\end{gathered}
$$

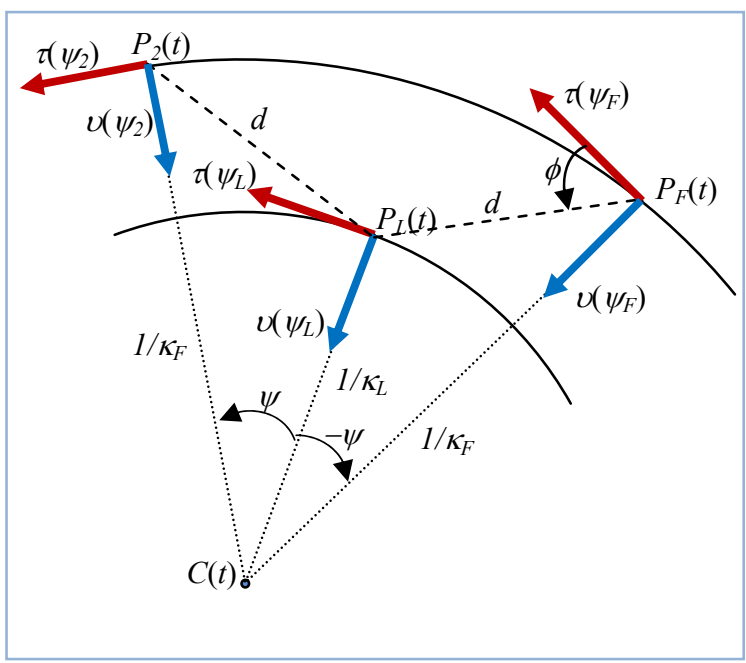

Figura 4. Formación estacionaria para $\boldsymbol{R}_{2}$ cuando

$$
\phi \geq 0 \text { y } \omega_{L}>0 \text {. }
$$

De los teoremas 3 y el 4 se deduce que si podemos construir un robot en formación estacionaria con $|\phi|<$ $\pi / 2$ entonces podemos construir un robot con formación estacionaria (24) o (25) según sea el signo de $\phi$.

NOTA 1. Un caso particular se tiene en el caso de que se fije la distancia a la que se quiere que el seguidor mantenga con respecto al líder y $\phi$ se determina de modo que se cumpla que $\kappa_{L}=\kappa_{F}$, es decir, el seguidor debe tener la misma velocidad angular que el líder y estar en la misma circunferencia siempre que $\kappa_{0} \neq 0$, se tiene entonces de (17): $\quad \psi_{L}-\psi_{F}=a \sin \left(\kappa_{L} d \cos \phi\right)=2 \phi, \quad$ luego $\sin \phi=\frac{\kappa_{L} d}{2}$, de lo que se siguen las relaciones

$$
\begin{aligned}
& \omega_{F}=2 v_{L} \frac{\sin \phi}{d} \\
& v_{F}=v_{L}
\end{aligned}
$$

\section{ALGORITMO DE CONTROL DE SEGUIMIENTO}

Para controlar el seguidor se procede de forma similar a [1] pero ahora no sólo cuando la trayectoria es una línea recta o una circunferencia, sino en todo punto, figura 2. Especificada una formación $(d, \phi)$ para todo $t$ se determina el único punto $P_{F}(t)$ en formación estacionaria con respecto al líder. Como hemos visto este punto se puede considerar situado en una cierta circunferencia siempre que el radio de curvatura del líder sea distinto de cero. A partir de $P_{F}(t)$ se determina un punto virtual $M(t)$ que en todo instante en el que $\kappa_{F}(t) \neq 0$ se encuentra en la misma circunferencia que $P_{F}(t)$ pero a una distancia $L>0$ por delante de él, siendo $L$ un parámetro de diseño. Por la nota 1 se sigue que en este caso el ángulo del vector velocidad de $P_{F}(t)$ con la línea de vista del punto virtual $M(t)$ es

$$
\sin \phi_{M}=\frac{\kappa_{F} L}{2}
$$

y el punto virtual se calcula como

$$
M(t)=P_{F}(t)+L \tau\left(\psi_{F}+\phi_{M}\right)
$$

La tangente a la circunferencia en $M(t)$ es

$$
\tau\left(\psi_{M}\right)=\tau\left(\psi_{F}+2 \phi_{M}\right)
$$

La ley de control del vehículo seguidor en una posición $P(t)$, figura (2) es

$$
\begin{gathered}
\dot{\psi}_{P}=-\frac{2 v_{P}}{L} \sin \eta, \quad|\eta| \leq \frac{\pi}{2} \\
\dot{\psi}_{P}=-\frac{2 v_{P}}{L} \operatorname{sign} \eta, \quad|\eta|>\frac{\pi}{2} \\
v_{P}=v_{M}-K\left(s_{1}+L \cos \beta\right)
\end{gathered}
$$

La velocidad angular del vehículo seguidor se calcula como en [1] pero la velocidad lineal se calcula ahora mediante la ecuación (32). El término $\left(s_{1}+L \cos \beta\right)$ determina la distancia de la coordenada $s_{1}$ actual a la proyección de $L$ sobre la línea de vista, es decir, la 
diferencia entre la distancia actual y la que tendría que tener si estuviera situado sobre el punto $P_{F}(t)$. Una vez que el ángulo se ha corregido mediante (8) la velocidad (9) hace que la distancia sea la adecuada.

Se puede demostrar que la ley de control propuesta converge a cualquier formación estacionaria que cumple las restricciones de los teoremas de la sección 3.

\section{EXPERIMENTO DE CONTROL DE FORMACIÓN}

Para validar los algoritmos se han realizado experimentos con dos modelos de barcos construidos en el Canal de Experiencias Hidrodinámicas del Pardo y que están descritos juntos con sus controladores en [1]. La figura 5 muestra los resultados de simulación cuando el barco líder realiza un camino representado por una lemniscata de Bernoulli de amplitud $200 \mathrm{~m}$, y centrada en $(30,-30)$. El barco líder lleva una velocidad de $2.5 \mathrm{~m} / \mathrm{s}$. Su posición inicial se toma como origen de coordenadas. $\mathrm{Su}$ rumbo inicial es de $0^{\circ}$. El barco seguidor está inicialmente en la posición $(-20,20)$ con rumbo inicial de $0^{\circ}$. La formación se especifica de modo que el barco seguidor lo hace a una distancia $d=20$ metros y un ángulo de vita de $+95^{\circ}$. Para la ley de guiado se escoge $L=45$ metros. La velocidad máxima del vehículo seguidor $6.25 \mathrm{~m} / \mathrm{s}$, y la mínima de $0.25 \mathrm{~m} / \mathrm{s}$. En la figura 5 a) se muestra la lemniscata en rojo, el barco líder en azul, y el barco seguidor en negro. La posición inicial de ambos se muestra mediante un círculo y una cruz del color del recorrido. Las líneas cian muestran el segmento que une la posición de ambos barcos en instantes de tiempo periódicos. Las figuras b) y c) muestran en línea continua azul la evolución de la distancia del barco seguidor al líder y del ángulo de vista, respectivamente. Las líneas discontinuas indican los valores de referencia. Tras el transitorio la distancia varía entorno al valor de referencia con una desviación máxima de $\pm 1 \mathrm{~m}$, y el ángulo de vista de $1.8^{\circ}$. La figura d) muestra los valores de curvatura del camino, en rojo, del vehículo líder en azul, y del vehículo seguidor en negro. Los valores de las curvaturas de los vehículos se calculan filtrando las arco tangentes de los vectores velocidad mediante la función de transferencia

$$
F(s)=\frac{s}{s+1}
$$

para aproximar la derivada de la arco tangente, y dividiendo la salida del filtro por la velocidad del vehículo. La figura e) muestra las señales de control del vehículo seguidor. La figura superior muestra la evolución de la velocidad lineal del vehículo y la parte inferior la velocidad angular.

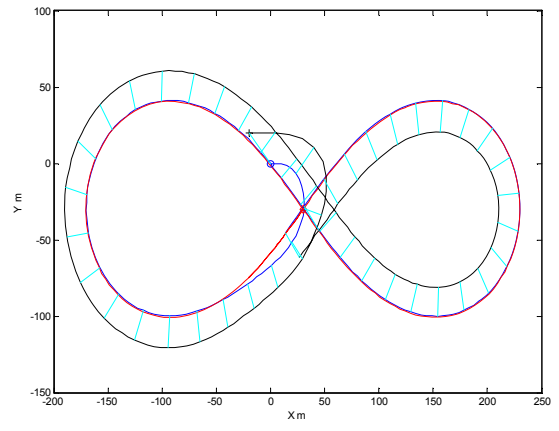

a)

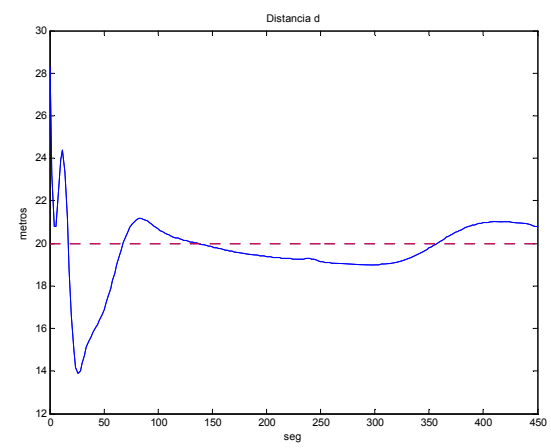

b)

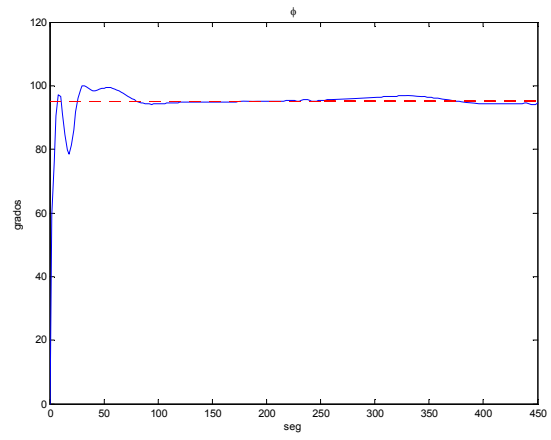

c)

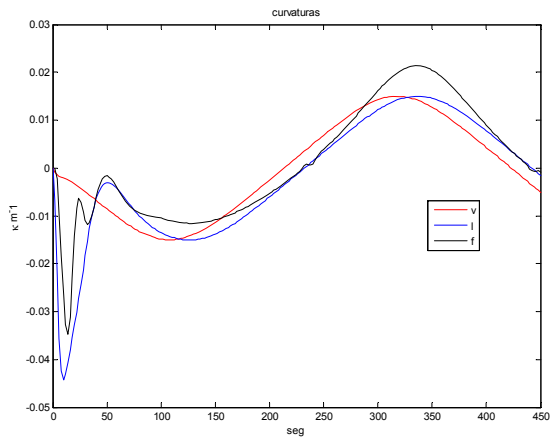

d) 

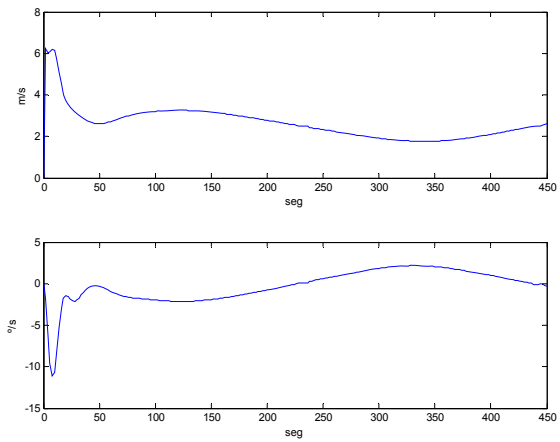

e)

Figura 5. Resultados obtenidos en el seguimiento de un vehículo que describa una lemniscata de Bernouilli.

\section{CONCLUSIONES}

Este trabajo presenta un nuevo algoritmo de control para formaciones y seguimiento de caminos para estructuras líder-seguidor. El algoritmo se basa en el concepto de formación estacionaria, lo que permite determinar un único punto para el seguidor a partir de la distancia deseada entre el seguidor y el líder, y el ángulo de vista desde el seguidor al líder, ángulo que puede variar entre menos pi y pi. El algoritmo tiene en cuenta las restricciones físicas del líder y el seguidor. Aunque se han mostrado ejemplos de aplicación para vehículos autónomos de superficie, el algoritmo se puede utilizar también en otros vehículos no holónomos.

\section{Agradecimientos}

Este trabajo ha sido financiado por los proyectos DPI2013-46665-C2-R1 y DPI2013-46665-C2-R2.

\section{Referencias}

[1] J.M. de la Cruz, J.A. López-Orozco, E. Besada-Portas, J. Aranda. Seguimiento de caminos para formaciones de vehículos marinos de superficie. Actas XXXV Jornadas de Automática, 3-5 sept. Valencia, 2014, pp. 588-595. CEA-IFAC.

[2] J. Manley. Unmanned surface vehicles, 15 years of development. Proc. MTS/IEEE Oceans'08, 1-4. 2008

[3] MTR, 2013. Unmanned Surface Vessels. Marine Technology Report, 24-35, May 2013.

[4] P. Svec, A. Thakur, E. Raboin, B. C. Shah, and S. K. Gupta. Target following with motion prediction for unmanned surface vehicle operating in cluttered environments. Auton. Robots, 36(4):383-405, 2014.

[5] H. Bai, M. Arcak, and J. Wen, Cooperative Control Design. Communications and Control Engineering, Springer, 2011.
[6] K. Pettersen, J. Gravdahl, and H. Nijmeijer, eds., Group Coordination and Cooperative Control. Springer, 2006.

[7] Das, A. K., Fierro, R., Kumar, V., Ostrowsky, J. P., Spletzer, J., \& Taylor, C. (2002). A vision-based formation control framework. IEEE Trans. on Robotics and Automation, 18(5), 813-825.

[8] L. Consolini, F. Morbidi, D. Prattichizzo, and M. Tosques. Leader-follower formation control of nonholonomic mobile robots with input constraints. Automatica, 44(5):1343-1349, 2008.

[9] M. Bibuli, M. Caccia, L. Lapierre \& G. Bruzzone, "Guidance of Unmanned Surface Vehicles. Experiments in Vehicle Following", IEEE Robotics \& Autom. Magazine, vol. 19, n. 3, pp. 92-102, 2012.

[10] E. Børhaug, A. Pavlov, E. Panteley, and K. Y. Pettersen, "Straight line path following for formations of underactuated marine surface vessels," Control Sys. Tech., IEEE Trans. on, vol. 19, no. 3, pp. 493-506, 2011.

[11] E. Peymani and T.I. Fossen, "Leader-Follower Formation of Marine Craft using Constraint Forces and Lagrange Multipliers," in Proc. 51st IEEE Conf. on Decision Control, pp. 2447-2452, Dec. 2012.

[12] R. Skjetne, S. Moi, and T. I. Fossen, "Nonlinear formation control of marine vessel," in Proc. Conf. Decision Control, 2002, pp. 1699-1704.

[13] M. Breivik, V. E. Hovstein, and T. I. Fossen, "Ship formation control: A guided leader-follower approach," IFAC World Congr., vol. 17, no. 1, pp. 16008-16014, Jul. 2008.

[14] E. Kyrkjebø, K. Y. Pettersen, M. Wondergem, and H. Nijmeijer, "Output synchronization control of ship replenishment operations: Theory and experiments," Control Eng. Pract., vol. 15, no. 6, pp. 741-755, Jun. 2006.

[15] L. Lapierre, D. Soetanto, and A. Pascoal. Nonlinear path following with applications to the control of autonomous underwater vehicles. In Decision and Control, 2003. Proceedings. 42nd IEEE Conference on, volume 2, pages 1256-1261. IEEE, 2003.

[16] M. Breivik, V. E. Hovstein, and T. I. Fossen. Straightline target tracking for unmanned surface vehicles. Modeling, Identification and Control, 29(4):131-149, 2008.

[17] J. de la Cruz, J. López-Orozco, E. Besada-Portas, and J. Aranda-Almansa. A streamlined nonlinear path following kinematic controller. In Robotics and Automation (ICRA), 2015 IEEE International Conference, pp. 6394-6401. IEEE, 2015.

[18] L. Lapierre and D. Soetanto. Nonlinear path-following control of an auv. Ocean Engineering, 34(11):17341744, 2007.

[19] S. Park, J. Deyst, and J. P. How. Performance and lyapunov stability of a nonlinear path following guidance method. Journal of Guidance, Control, and Dynamics, 30(6):1718-1728, 2007. 